\title{
Features of conduction electrons motion in the field of coherent light beams
}

\author{
O.Yu. Semchuk ${ }^{*}$, M. Willander ${ }^{* *}$, and M. Karlsteen ${ }^{* *}$ \\ "Institute of Surface Chemistry of the National Academy of Sciences of Ukraine, \\ 17 General Naumov Street, 03164, Kyiv, Ukraine \\ ${ }^{* *}$ Department of Physics, Chalmers University of Technology and Göteborg University, \\ S-41296, Göteborg, Sweden
}

\begin{abstract}
Conduction electrons moving in a non-homogeneous field of coherent light beams (CLB) are investigated. It is shown that a conduction electron simultaneously takes part in two oscillations. More exactly, an electron oscillate with the frequency $\omega$ and in addition, it carry out a forced oscillation with the frequency $\omega_{z}<<\omega$ ( $\omega$ is the CLB frequency) inside a one-dimensional potential well that is induced in the semiconductor by the CLB.
\end{abstract}

Keywords: semiconductors, conduction electrons, coherent light beams, electronic transport, electron motion, frequency, potential well, force.

Paper received 13.12.00; revised manuscript received 23.01.01; accepted for publication 16.02.01.

\section{Introduction}

It is well known in solid-state physics that for a spatially periodic Hamiltonian, a quasi-momentum, and corresponding Bloch wave functions exists [1]. Analogously, for a periodic time dependent Hamiltonian of the crystal, one expects existence of quasi-energies and the Blochtype states defined by the term «steady states» [2,3]. Such steady states are used, for example, in the theory of susceptibility [4,5], in the theory of multiple-quantum transitions for discrete levels [6,7], in the theory of kinetic phenomena in the field of intense electromagnetic wave $[8,9]$. In these theories considered were only periodic time dependencies of electromagnetic wave vector potential. The spatial period of this potential is neglected. Therefore, it would not be correct to consider various phenomena produced in solids by intense coherent laser beams (CLB). In the present paper, an investigation on how a high frequency CLB field influences on the motion of conduction electrons in solid state is carried out. It is shown that a force acts on the conduction electrons in the field of CLB, which implies that quasi-energy of an electron becomes a function of coordinate in this case. The high-frequency non-homogeneous field of CLB, inside of semiconductor bulk, creates periodic one-dimensional potential wells for conduction electrons moving along the $\mathrm{OZ}$ axis. For that reason the conduction electron take part simultaneously in two motions stimulated by the CLB field. Namely, it oscillates with the frequency $\omega$ in the field of CLB and, in addition, it performs a forced oscillation with the frequency $\omega_{z} \ll \omega$ ( $\omega$ is the CLB frequency) inside the one-dimensional well induced by the CLB.

\section{Conduction electrons motion in the field of CLB}

Let's consider the motion of conduction electron in an infinite wide conduction-band. The CLB vector potential is given as

$$
\vec{A}(\vec{r}, t)=\sum_{j} \vec{A}_{j} \operatorname{Cos}\left(\omega t-\vec{k}_{j} \vec{r}-\varphi_{j}\right),
$$

where frequency $\omega$ satisfies the condition $\omega \tau>>1$ ( $\tau$ is electron collision time).

Now, we write down the non-relativistic classical equation of motion of the conduction electrons in a CLB field

$$
m \frac{d^{2} \vec{r}}{d t^{2}}=e \vec{E}(\vec{r}, t)+\frac{e}{c} \frac{d \vec{r}}{d t} \times \vec{H}(\vec{r}, t)
$$

where $\vec{E}(\vec{r}, t)$ and $\vec{H}(\vec{r}, t)$ are CLB electric and magnetic fields strengths, $\vec{v}=\frac{d \vec{r}}{d t}$ is the initial velocity of electron. Taking $\vec{r}(t)$ in the form 
$\vec{r}(t)=\vec{R}(t)+\vec{\rho}(t)$,

where $\vec{R}(t)$ is a function that changes slowly during CLB field action, and $\vec{\rho}(t)$ is an oscillating additive, that is considered further on to being a small parameter. Now we rewrite (3) as

$$
\begin{aligned}
& \frac{d^{2} \vec{R}}{d t^{2}}+\frac{d^{2} \vec{\rho}}{d t^{2}}=\frac{e}{m} \vec{E}(\vec{R}+\vec{\rho}, t)+ \\
& +\frac{e}{m c}\left\{\left[\frac{d \vec{R}}{d t}, \vec{H}(\vec{R}+\vec{\rho}, t)\right]+\left[\frac{d \vec{\rho}}{d t}, \vec{H}(\vec{R}+\vec{\rho}, t)\right]\right\} .
\end{aligned}
$$

Than we expand $\vec{E}(\vec{r}, t)$ and $\vec{H}(\vec{r}, t)$ into Taylor's series with respect to the small parameter $\vec{\rho}$

$$
\begin{aligned}
& \vec{E}(\vec{R}+\vec{\rho}, t)=\vec{E}(\vec{R}, t)+\vec{\rho} \frac{\partial \vec{E}(\vec{R}, t)}{\partial \vec{R}}+\frac{1}{2} \vec{\rho}^{2} \frac{\partial^{2} \vec{E}(\vec{R}, t)}{\partial \vec{\rho}^{2}}+\ldots \\
& \vec{H}(\vec{R}+\vec{\rho}, t)=\vec{H}(\vec{R}, t)+\vec{\rho} \frac{\partial \vec{H}(\vec{R}, t)}{\partial \vec{R}}+\frac{1}{2} \vec{\rho}^{2} \frac{\partial^{2} \vec{H}(\vec{R}, t)}{\partial \vec{\rho}^{2}}+\ldots
\end{aligned}
$$

Due to restrictions to the lowest order of the infinitesimal we write (4) in the form:

$$
\begin{aligned}
& \frac{d^{2} \vec{R}}{d t^{2}}+\frac{d^{2} \vec{\rho}}{d t^{2}}=\frac{e}{m}\{\vec{E}(\vec{R}, t)+\vec{\rho} \nabla \vec{E}(\vec{R}, t)\}_{+} \\
& +\frac{e}{m c}\left\{\begin{array}{l}
{\left[\frac{d \vec{R}}{d t}, \vec{H}(\vec{R}, t)\right]+\left[\frac{d \vec{\rho}}{d t}, \vec{H}(\vec{R}, t)\right]+} \\
{\left[\begin{array}{l}
\left.\frac{d \vec{R}}{d t}, \vec{\rho} \nabla \vec{H}(\vec{R}, t)\right]+\left[\frac{d \vec{\rho}}{d t}, \vec{\rho} \nabla \vec{H}(\vec{r}, t)\right]
\end{array}\right\} .}
\end{array}\right.
\end{aligned}
$$

We can solve equation (6) by the iteration approximation method, assuming that $|\vec{E}| \gg|\vec{H}|$. First, put down the equation in the first approximation

$$
\frac{d^{2} \vec{\rho}}{d t^{2}}=\frac{e}{m} \vec{E}(\vec{R}, t) \text {. }
$$

Integration of (7) gives:

$$
\begin{aligned}
& \frac{d \vec{\rho}}{d t}=-\frac{e}{m \omega} \sum_{j} \vec{E}_{j} \cos \left(\omega t-\vec{k}_{j} \vec{R}-\varphi_{j}\right), \\
& \vec{\rho}=-\frac{e}{m \omega^{2}} \vec{E}(\vec{R}, t) .
\end{aligned}
$$

Now, we rewrite (6) with respect (7) and (8) in the form

$$
\frac{d^{2} \vec{R}}{d t^{2}} \approx-\frac{e^{2}}{2 m^{2} \omega^{2}} \nabla \vec{E}^{2}(\vec{R}, t)
$$

Averaging the right-hand side of (9) over the CLB field period gives the following equation, which describes the electron motion in the average CLB field

$$
m \frac{d^{2} \vec{R}}{d t^{2}}=-\frac{e^{2}}{4 m c^{2}} \nabla \sum_{j j^{\prime}} \vec{A}_{j} \vec{A}_{j^{\prime}} \operatorname{Cos}\left[\left(\vec{k}_{j}-\vec{k}_{j^{\prime}}\right) \vec{R}+\varphi_{j}-\varphi_{j^{\prime}}\right]
$$

Thus, it follows from (10) that the CLB high-frequency electromagnetic field acts on the electrons with the force

$$
\vec{F}=-\frac{e^{2}}{4 m c^{2}} \frac{\partial}{\partial \vec{R}}\left(\sum_{j j^{\prime}} \vec{A}_{j} \vec{A}_{j^{\prime}} \operatorname{Cos}\left[\left(\vec{k}_{j}-\vec{k}_{j^{\prime}}\right) \vec{r}+\varphi_{j}-\varphi_{j^{\prime}}\right]\right),
$$

The expression for this force was obtained earlier by one of the authors [11] using standard method a quantum transport equations for the quantum-mechanic operators for the construction of the quantum kinetic equations for electrons and magnons in the field of CLB.

Now we shall analyze the character of electron motion in a field of CLB in more details. With this aim, we shall re-write (11) as:

$\frac{d^{2} \vec{R}(t)}{d t^{2}}=-\nabla \Phi$,

where $\Phi=\frac{\Delta E_{\vec{A}_{j}} \vec{A}_{j^{\prime}}}{m}$ has the meaning of the CLB highfrequency potential, which is entered for the description the average motion at a charge in non-homogeneous electric field [10];

$\Delta E_{\vec{A}_{j} \vec{A}_{j^{\prime}}}=\frac{e^{2}}{4 m c^{2}} \sum_{j j^{\prime}} \vec{A}_{j} \vec{A}_{j^{\prime}} \operatorname{Cos}\left[\left(\vec{k}_{j}-\vec{k}_{j^{\prime}}\right) \vec{R}+\varphi_{j}-\varphi_{j^{\prime}}\right]$ is

the mean value of quasi-energy of conduction electrons in the field of CLB.

Thus, the action of a non-homogeneous CLB field on the motion of conduction electrons can be presented as the result of an effective potential field. The potential of this effective field is equal to $\Delta E_{\vec{A}_{j} \vec{A}_{j^{\prime}}} / m$. It means that conduction electrons in the field of CLB a force acts as $\vec{F}=-\operatorname{grad}\left(\Delta E_{\vec{A}_{j} \vec{A}_{j^{\prime}}}\right)$, caused by the coordinate dependence of the main value of quasi-energy of conduction electron in the field of CLB. Note, that in the spatial homogeneous field of an electromagnetic wave, when the amplitude of its electrical field $\vec{E}$ does not depend on coordinate $\vec{r}\left(\vec{E}(t)=\vec{E}_{0} \sin \omega t\right)$, quasi-energy of conduction electrons is equal to

$\Delta E_{\vec{A}_{j} \vec{A}_{j^{\prime}}}=\Delta E=\frac{e^{2} E_{0}^{2}}{4 m \omega^{2}}$.

Thus, in this case, quasi-energy of conduction electrons is connected only with the spatial homogeneous field of electromagnetic wave, which produces only a shift of 
electron energy spectrum as whole on the quantity $\Delta E=e^{2} E_{0}^{2} /\left(4 m \omega^{2}\right)$ [8]. It is possible to specify some such cases when the calculation of a value $\Delta E$ is necessary:

1. The system contains some types of carriers with different charges or effective masses. In this case for each type of carriers there will be a shift $\Delta E$ that results a change of distance between appropriate branches of a spectrum.

2. The initial energy spectrum is non-parabolic, i.e. electron effective mass have different values in different parts of a conductivity band.

3. The conduction electrons mechanical trajectory in the field of CLB

Consider the instance where the outer surface $z=0$ of semiconductor is exposed to two symmetrically oriented CLB that converge in the bulk of the semiconductor at a small angle $2 v$ and whose vector-potential is given by the following expression (Fig. 1)

$$
\begin{aligned}
& \vec{A}(\vec{r}, t)=\vec{A}_{1} \cos \left(\omega t-k_{x} x-k_{z} z-\varphi_{1}\right)+ \\
& +\vec{A}_{2} \cos \left(\omega t-k_{x} x+k_{z} z-\varphi_{1}\right), \quad \vec{A} \| O Y .
\end{aligned}
$$

Received as a result of an interference between these two CLB, the interference picture with good precision can be approximated by a standing laser wave with the spatial period $L=\lambda_{0} / 2 \sin \vartheta\left(\lambda_{0}\right.$ is the CLB wavelength in the semiconductor bulk) [12]. Thus, in the bulk of semiconductor, CLB may be approximated by the laser standing wave

$$
\vec{E}=\vec{E}_{0} \sin \left(k_{z} z\right) \sin (\omega t)
$$

where $\vec{E}_{0}=\frac{\omega}{c} \vec{A}_{1} \approx \frac{\omega}{c} \vec{A}_{2}$ is the average electric field intensity of the standing laser wave (CLB).

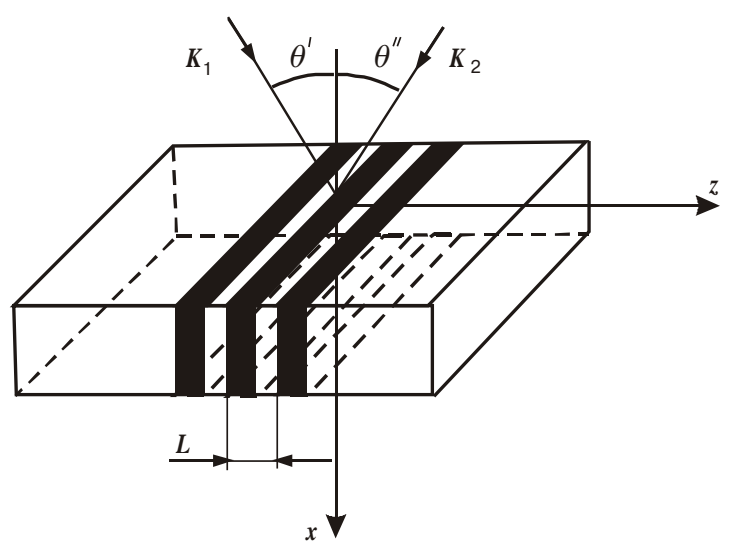

Fig. 1. Interference scheme for two CLB illuminating the front surface $z=0$ of a semiconductor sample. $\left(L=\lambda_{0} / 2 \sin \vartheta\right.$ is the period of interference picture).
The high-frequency potential $W$ corresponding to the interference picture in the bulk of semiconductor, produced by CLB, is equal to

$W \approx\left(\frac{e E_{0}}{2 m \omega}\right)^{2} \sin ^{2} k_{z} z$

In Fig. 2 the structure of dimensionless high-frequency potential $W / W_{0}\left(W_{0}=\left(\frac{e E_{0}}{2 m \omega}\right)^{2}\right)$ is shown. Therefore, the high-frequency potential $W$ may be considered as a periodic potential well for the conduction electrons moving along the $\mathrm{OZ}$ axis in the inside of semiconductor bulk.

Let a conduction electron, which is moving with velocity $v_{0}\left(v_{0}=\sqrt{2 \frac{e}{m} V_{0}}\right.$ is the initial velocity of conduction electron in the point $z=0$ ) along $\mathrm{OZ}$ axis could be located inside of a potential well, created by CLB. The fulfillment of the following condition is necessary

$\frac{e}{m} V_{0}<W$

which, together with the condition $|\vec{\rho}|<<L$, imposes the a restrictions of the amplitude of an electric field of CLB, creating a potential well

$2 \omega \sqrt{\frac{m}{e} V_{0}}<E_{0}<<\frac{m}{e} \omega^{2} L$

Thus, if at the center of a potential well has $E_{0}=0$, conduction electron with energy equal $e V_{0}$ is located in-

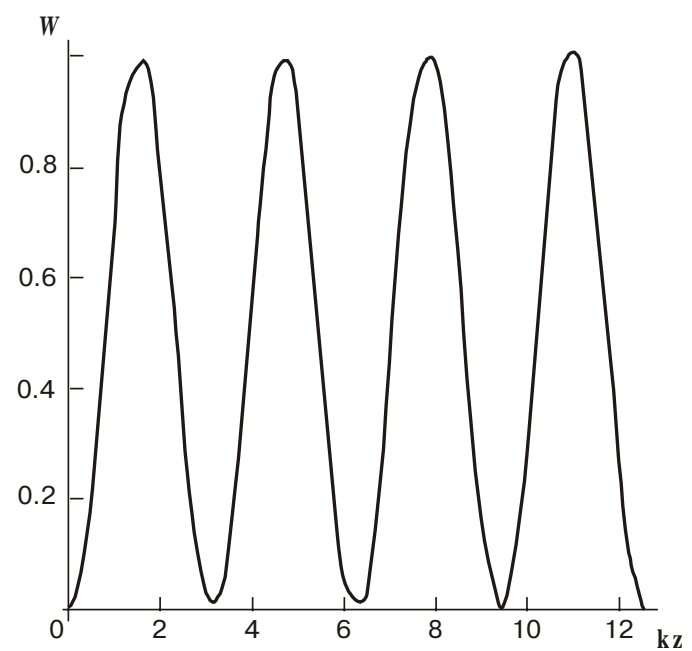

Fig. 2. The structure of dimensionless high-frequency potential $W\left(W / W_{0}, W_{0}=\left(\frac{e E_{0}}{2 m \omega}\right)^{2}\right)$ as a function of the dimensionless
coordinate $k z$. 
side the potential well, in which the boundary conditions (17) are valid.

Now we shall consider a movement of a conduction electron inside of the potential well in more details. Substituting (14) and (15) into (8) and (9) we obtain the following equations for determining $z$ and $x$

$$
\frac{d^{2} z}{d t^{2}}+\omega_{z}^{2} z=0, \quad x=-\frac{\sqrt{2} z}{\omega \omega_{z}} \sin \omega t
$$

where $\omega_{z}=\frac{e E_{0}}{\sqrt{2} m c}$ is the electron oscillation frequency inside the one-dimensional potential well, produced by CLB.

The first of the equations under the number (18) is the free electron harmonic oscillation one. Its solution is

$$
z=C_{1} \cos \omega_{z} t+C_{2} \sin \omega_{z} t
$$

and with accounting the initial conditions $C_{1}=0$, $C_{2}=\frac{v_{0}}{\omega_{z}}$ has the form

$z=\frac{v_{0}}{\omega_{z}} \sin \omega_{z} t$

Making use of (20), we write down equations, which describe the conduction electron mechanical trajectory as:

$z=\frac{v_{0}}{\omega_{z}} \sin \omega_{z} t, \quad x=-\frac{v_{0}}{\omega} \sqrt{2} \sin \left(\omega_{z} t\right) \sin (\omega t)$.
In Fig. 3, the conduction electron mechanical trajectories in a field of CLB for the following meanings of parameters: $\frac{\omega}{\omega_{z}}=10, \quad \frac{\omega}{\omega_{z}}=11, \quad \frac{\omega_{z} z_{\max }}{c}=1, \quad z>0$ are shown.

Depending on a ratio between frequency of a CLB field $\omega$ and a frequency of oscillations of conduction electrons inside a potential well $\omega_{z}$, the conduction electrons mechanical trajectories will be various. From

Fig. 3a, for example, when $\frac{\omega}{\omega_{z}}=10$, follows that the conduction electron mechanical trajectory inside a potential well will be made. It means that the electron alternately being reflected from walls of a potential well will make inside oscillations it with frequency is follows $\omega_{z}$.

From Fig. $3 b$ is follows that in a case when $\frac{\omega}{\omega_{z}}=11$, that the conduction electron mechanical trajectory will be open. It means, that having made some oscillations inside of a potential well the electron can abandon and further move only under the activity of the field of CLB.

Thus, the electron in the field of CLB takes part simultaneously in two motions. Namely, it oscillates with the frequency $\omega$ and amplitude $\vec{a}=\frac{e \vec{E}_{0}}{m \omega^{2}}$ in the field of CLB and performs forced oscillations at the frequency $\omega_{z}<\omega \omega$ inside of a one-dimensional well, produced along $\mathrm{OZ}$ axis by CLB.

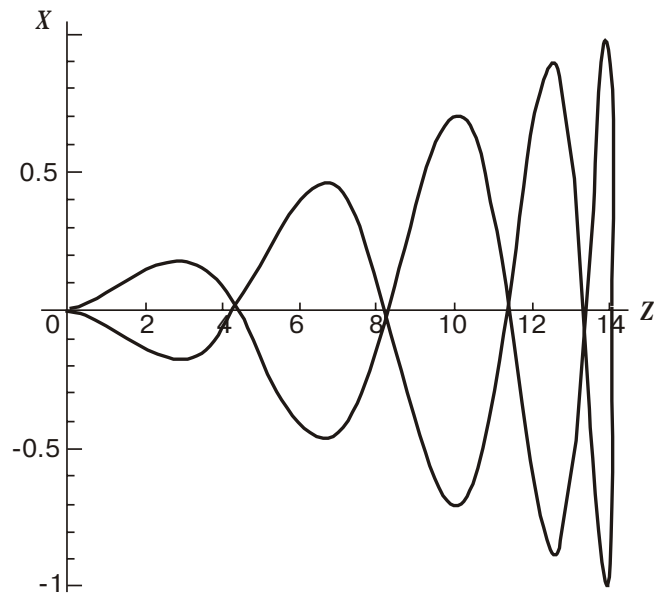

a)

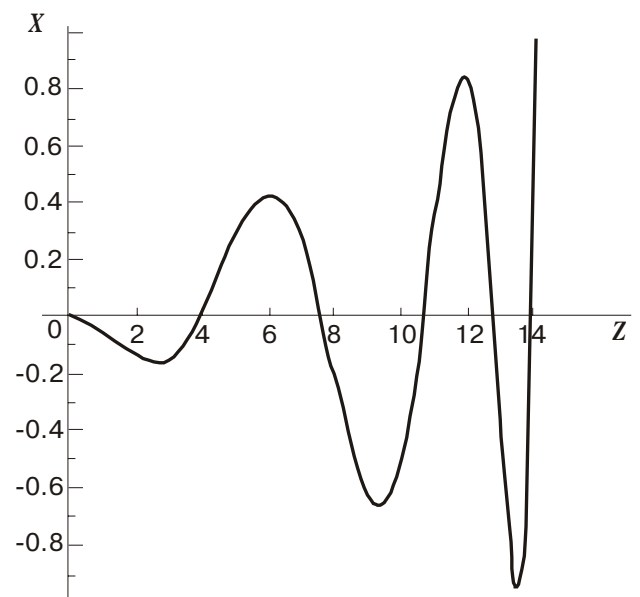

b)

Fig. 3. The conduction electrons mechanical trajectories in a field of CLB for the following meanings of parameters: a $-\frac{\omega}{\omega_{z}}=10$, $\frac{\omega}{\omega_{z}}=10, \quad \frac{\omega_{z} z_{\max }}{c}=1, \quad z>0 ; \quad b-\frac{\omega}{\omega_{z}}=11, \quad \frac{\omega_{z} z_{\max }}{c}=1, \quad z>0$. 


\section{Conclusions}

The above reported results enable us to make the following conclusions:

1. The action of an inhomogeneous CLB field on the motion of conduction electrons can be presented as the result of an effective potential field. The potential of this effective field is equal to $\Delta E_{\vec{A}_{j}} \vec{A}_{j^{\prime}} / m$. This means that on conduction electrons in the field of CLB are acts the force $\vec{F}=-\operatorname{grad}\left(\Delta E_{\vec{A}_{j}} \vec{A}_{j^{\prime}}\right)$ caused by that the quasi-energy of the conduction electrons in the field of CLB becomes a function of coordinate $\vec{r}$.

2. The high-frequency non-homogeneous field of CLB produced inside of a semiconductor bulk creates periodic one-dimensional potential wells for conduction electrons moving along the $\mathrm{OZ}$ axis.

3. In the field of CLB, conduction electrons take part simultaneously in two motions. Namely, they oscillate with the frequency $\omega$ and amplitude $\vec{a}=\frac{e \vec{E}_{0}}{m \omega^{2}}$ in the field of CLB and perform forced oscillations at the frequency $\omega_{z}<<\omega$ inside a one-dimensional well induced by CLB.

\section{Acknowledgments}

One of authors (Dr. O.Yu. Semchuk) thanks the Royal Swedish Academy of Sciences and Swedish Natural Science Research Council (NFR) for financial support for fulfillment of this work.

\section{References}

1. Kittel C, Quantum Theory of Solids, Willey, New York, 1987.

2. Hideo Sambe. Steady States and Quasi-energies of a Quantum-Mechanical System in an Oscillating Field // Phys.Rev, A7(6), pp. 2203-2213 (1973).

3. Ya. B. Zeldovich, Dispersion and radiation by quantum system in a strong electromagnetic wave // Sov. Phys. Uspekh, 14(1), pp. 139-151 (1973).

4. R.H. Young, W.J. Deal, Jr. and N.P. Kesther, Quasi-periodic states of an oscillation Hamiltonian // Mol.Phys, 17, pp. 369-375 (1969).

5. P.W. Langhoff, S.T.Epstein and M.Karplus, Aspects of timedependent perturbation theory// Rev.Mod.Phys, 44(3), p. 602 (1972).

6. J.H. Shirley, Solution of Shrodinger equation with a Hamiltonian periodic in time// Phys. Rev, 138, p. 8974-80 (1965).

7. W.W. Hisks, R.A. Hess, and W.S. Cooper, Combined Zeeman and high-frequency Stark effects with applications to neutral-helium lines useful in plasma diagnostics// Phys.Rev, A5(3), pp. 490-507 (1972).

8. V.P. Seminozhenko, Kinetics of interacting quasiparticles in strong external fields// Phys. Rep, 91(3), pp.103-181 (1982).

9. V.P. Seminoshenko and A.A. Yatsenko, Kinetic equations for electrons and phonons in a strong constant electric field // Phys. Letters, 75A(4), pp. 267-268 (1980).

10. Gaponov A.V. and. Miller M.A., Potential wells for charger particles in a high-frequency electromagnetic field //Sov. Phys. JETP, 7(1), pp.168-169 (1958).

11. Semchuk O.Yu., Grechko L.G. and Ogenko V.M., Field influence on the parameters of ferromagnetic semiconductor superlattices formed by coherent light beams// Phys Stat Sol (b), 157, pp. 451-458 (1990).

12. V.L. Vinetskii, N.V. Kukhtarev, S.G. Odulov and M.S. Soskin, Dynamic self-diffraction of coherent light beams // Sov. Phys. Usp., 22(9), pp.742-756 (1979). 